\title{
Extracellular Matrix
}

National Cancer Institute

\section{Source}

National Cancer Institute. Extracellular Matrix. NCI Thesaurus. Code C13232.

The extracellular matrix is a network of fibers that hold cells together. It is found within the extracellular space and in association with the basement membrane of the cell surface. It promotes cellular proliferation and provides a supporting structure to which cells or cell lysates in culture dishes adhere. It is broken down during normal physiological and disease processes such as bone remodeling, embryogenesis, cancer, and arthritis. 